\title{
Design of a Parking Violation Penalty Equipment
}

\author{
Jiawang $\mathrm{CAO}^{1, \mathrm{a}}$, Xiangdong $\mathrm{YOU}^{2, \mathrm{~b}}$, Cheng $\mathrm{DA}^{3, \mathrm{c}}$, Yifei WANG ${ }^{4, \mathrm{~d}}$, Mengyuan \\ ZHANG ${ }^{5, \mathrm{e}}$ \\ ${ }^{1}$ School of Electronic Engineering, Beijing University of Posts and Telecommunications, Beijing, \\ 100876, China \\ ${ }^{2}$ School of Information and Communication Engineering, Beijing University of Posts and \\ Telecommunications, Beijing, 100876, China \\ ${ }^{3}$ School of Electronic Engineering, Beijing University of Posts and Telecommunications, Beijing, \\ 100876, China \\ ${ }^{4}$ School of Electronic Engineering, Beijing University of Posts and Telecommunications, Beijing, \\ 100876, China \\ ${ }^{5}$ School of Electronic Engineering, Beijing University of Posts and Telecommunications, Beijing, \\ 100876, China \\ aemail: mr_jwcao@163.com, bemail: youxiangdong@139.com, cemail: \\ 645398408@qq.com, demail: cynthia_fifi@bupt.edu.cn, eemail: myzhang@bupt.edu.cn
}

Keywords: Parking Violation Penalty Equipment; License Plate Recognition; Bluetooth Printing

\begin{abstract}
This paper introduces a design of a parking violation penalty equipment which aims to deal with violation of parking information. The design uses the equipment to take photos for parking violation. The equipment identifies the license plate number and fills in the license plate information automatically. It also can obtain the location of illegal sites based on GPS data automatically and uses Bluetooth printer to print a parking ticket. Then it uploads to server via the mobile network. People can query history through the server. It can reduce the penalty time to improve the work efficiency.
\end{abstract}

\section{Introduction}

Nowadays, most parking tickets are written manually by the law enforcement officer. On the site of violation, traditional way to record information is to use papery ticket. There are often two traffic assistants on site. The one takes photos and another fills in tickets. It's inefficient and erroneous to accomplish penalty. When tickets return to Traffic Administration Bureau, one person will be needed to input data manually. Therefore, it's an urgent question to save the human cost and improve work efficiency.

The article realizes a method to deal with violation of parking information rapidly. The method uses an ordinary intelligent terminal, such as smartphone and pad. Fast recording, completing penalty and printing tickets are available by means of a special software in terminal. This method makes penalty not only more reasonable, but also efficient because only one person is needed on the spot.

The article designs and realizes taking photos of violation, license plate recognition, automatic positioning, automatic writing ticket and printing tickets on the spot by Bluetooth printer.

\section{Operating principle of an equipment}

Workflow. When a traffic assistant arrives the site, he starts the software to record information, to obtain the location, to identify the system time, to take photos for license-plate, to recognize license-plate. Then he saves all information and uses Bluetooth printer to print tickets pasted on the car to notice the owner.

S1: Starting the special software in intellectual terminal to record violation when arrival.

S2: Taking photos for violation, license plate recognition, automatic orientation, identifying the system time, filling other information in ticket automatically. 
S3: Generating the electronic tickets, printing the paper tickets, uploading the information, saving in database via the server.

S4: Querying violation information

The workflow of software in equipment from S1 to S4 is shown in Fig.1.

S1:Starting the special software in intellectual terminal to record violation when arrival.

Thing photos for violation, license plate recognition, automatic

orientation, identifying the system time, filling other informations in ticket automatically.

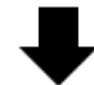

S3:Generating the electronic tickets, printing the paper tickets, uploading the information, saving in datebase via the server.

\section{S4:Query violation information}

Fig.1 The workflow of software in equipment

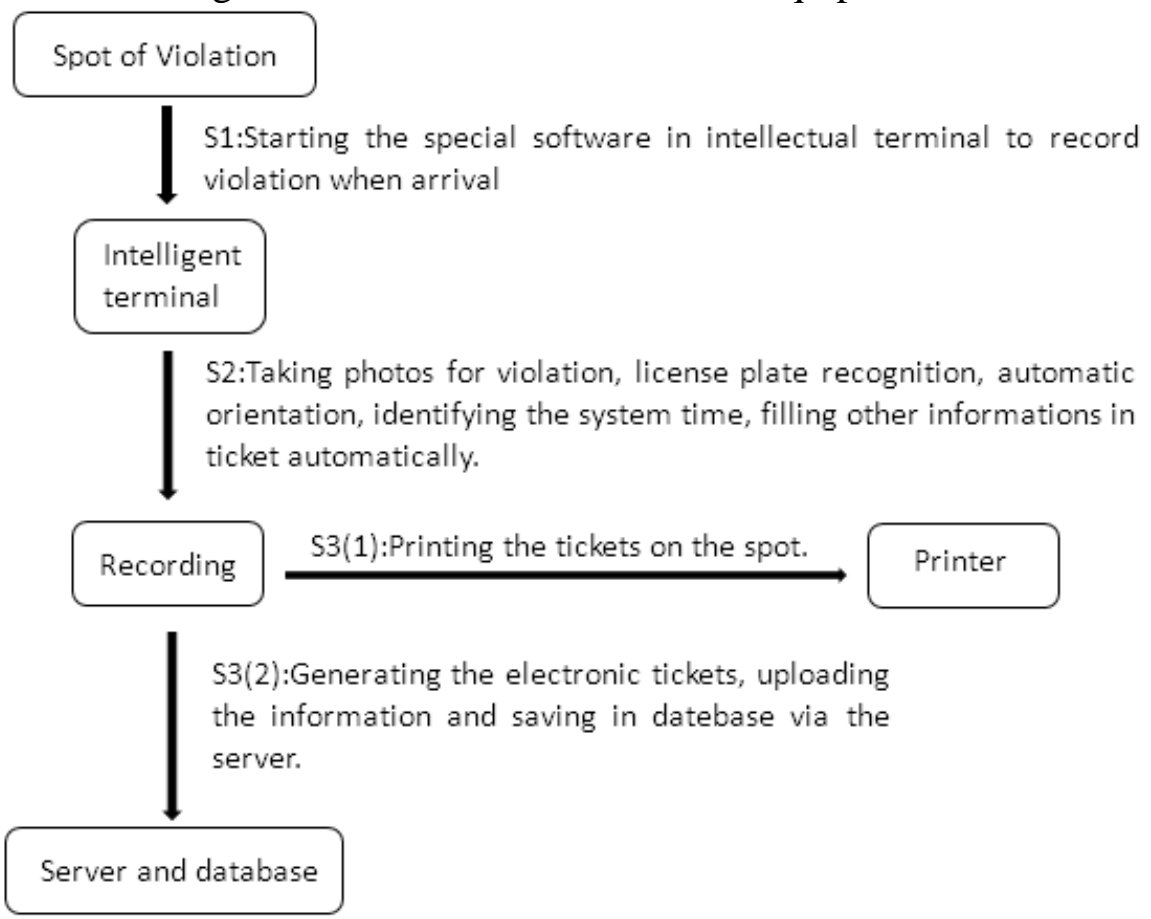

Fig.2 Constitution of parking violation penalty system

Constitution of parking violation penalty system. The parking violation penalty system includes penalty equipment based on intelligent terminal, Bluetooth printer and background server. A special software is installed in intelligent terminal to collect penalty information rapidly. Bluetooth printer receives printer orders and contents from the equipment and prints tickets. Background server receives penalty information uploaded by the equipment and provides inquiry service. The server programmed by PHP uses Apache as web server. MySQL serves as database to save penalty 
information so that administrative staff and drivers can query history. The whole constitution is as shown in Fig.2.

\section{The research and realization of technique}

Automatic license plate recognition. The license number is extracted from a car photo by using pattern recognition. Tesseract OCR is adopted after comparing with the openCV(open source computer vision library). There are three reasons.

(1) OpenCV needs training date by ourselves therefore quantity of sample needed is numerous and difficulty is high[1]. By contrast, Tesseract OCR can be used directly by downloading trained Android data package[2].

(2) The template matching is inaccurate.

(3) The disadvantage of Tesseract OCR is that recognition process is uncontrollable[3].

Automatic orientation. LBS application based on mobile client provides basic positioning ability. Using GPS, base station,WIFI to obtain violation spot name automatically via API provided by Baidu map. When application makes a request to positionning SDK, SDK will order by the situation of positioning factor(GPS, base station, WIFI) to produce result. If user sets GPS is prior, GPS is supreme. Then SDK return results according to other factors. They are shown in Fig.3.

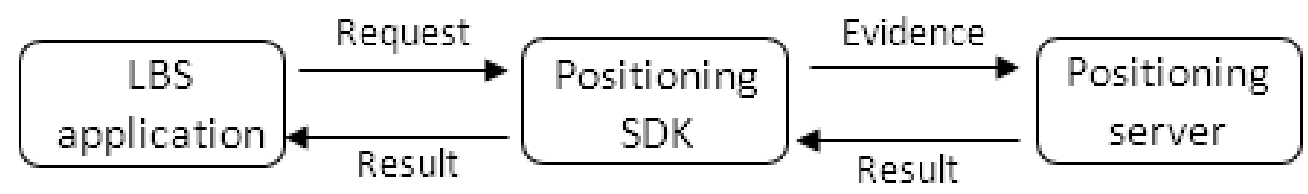

Fig.3 Principle of positioning automatic

Storage in equipment based on Android. Penalty information on the spot is saved by SQLite, including vehicle type and color, license plate number and color, simple description, information of traffic assistants, photos on the spot.

Bluetooth printing. There are two questions in printing black-and-white photograph using thermosensitive printer.

(1) Printing photo is different from printing text because of binaryzation. Then binary data is converted to data stream and sent to printer.[4]

(2) When it comes to printing, the width is restricted in order to prevent messy code or image error caused by exceeding paper scope.

Cross-platform file transfer. File is transfered from Android to PC by uploading to server through HTTP so that data administration and query. JSON(JavaScript Object Notation) is adopted after studying all kinds of coded format to solve problem of cross-platform transfer files[5].

(1) Firstly, Bandwidth of JSON is less because of compressed format.

(2) Secondly, JSON is easy to be read by client.

(3) Thirdly, JSON supports various languages, such as PHP, C, C++.

\section{Application software based on Android}

Software process. The whole software process is as shown in Fig.4 from main interface to each sub-function. 


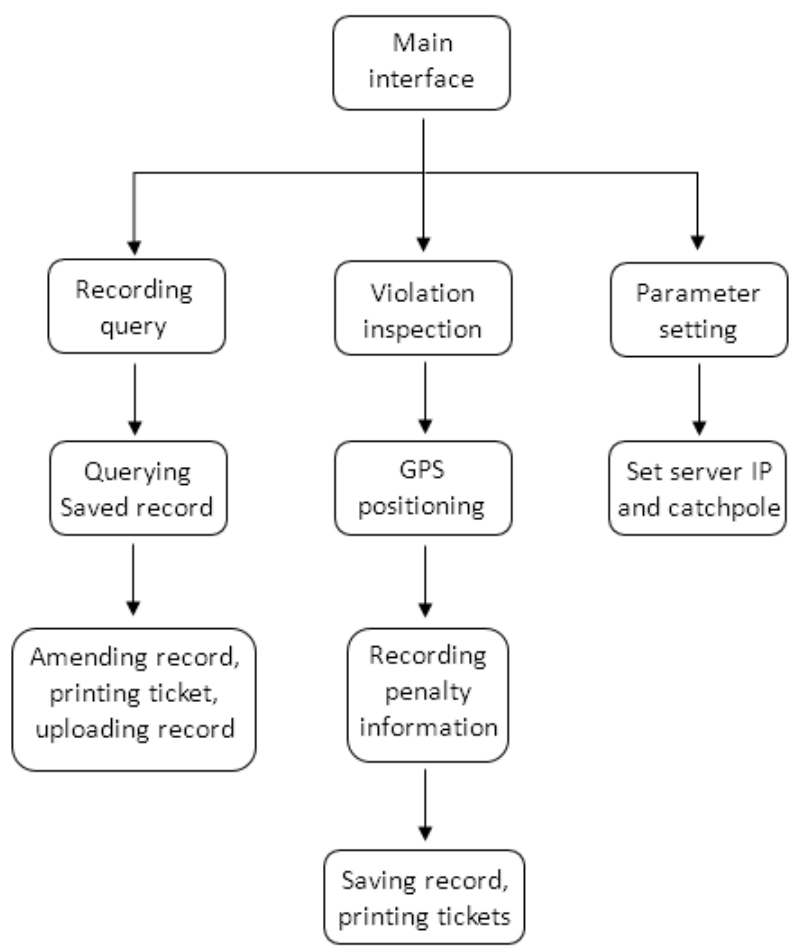

Fig.4 Software process

Software GUI. GUI is consist of recording query, violation inspection and parameter setting.

- Main UI interface is in Figure 5.

- In recording query, there are query, emendation, saving and printing shown in Fig.6.

- In violation inspection, there are vehicle type and color, license plate number and color, simple description, information of traffic assistants, photos on spot shown in Fig.7

- In parameter setting, there are server IP setting and catchpole name setting shown in Fig.8.

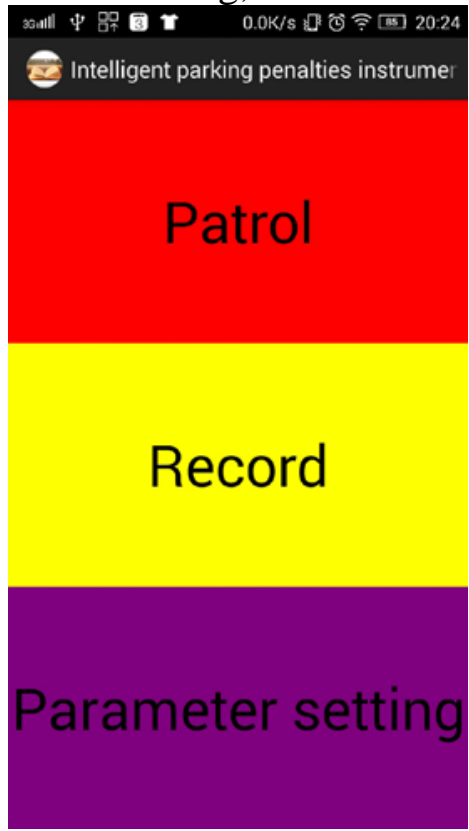

Fig.5 Main UI interface

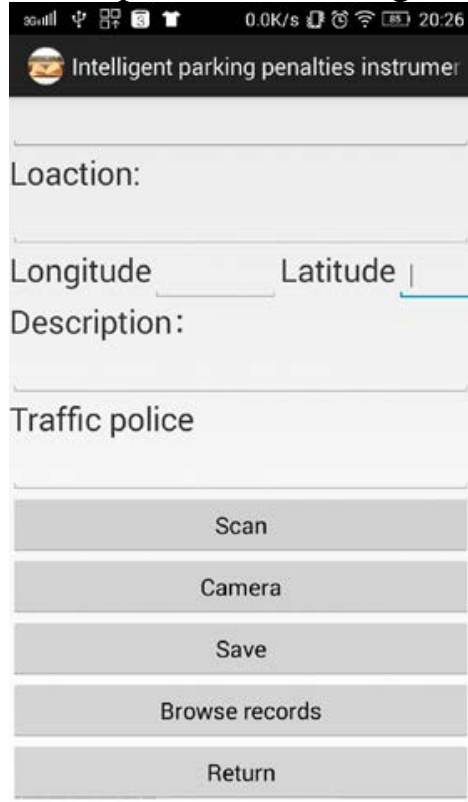

Fig.6 Recording query 


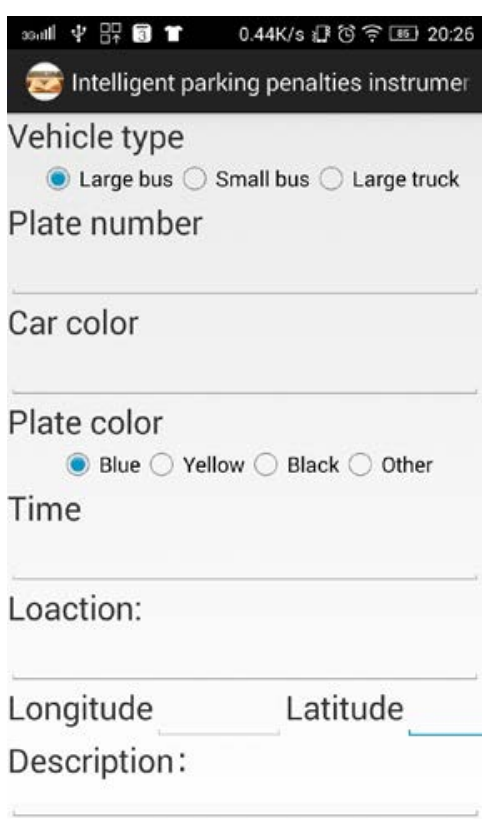

Fig.7 Violation inspection

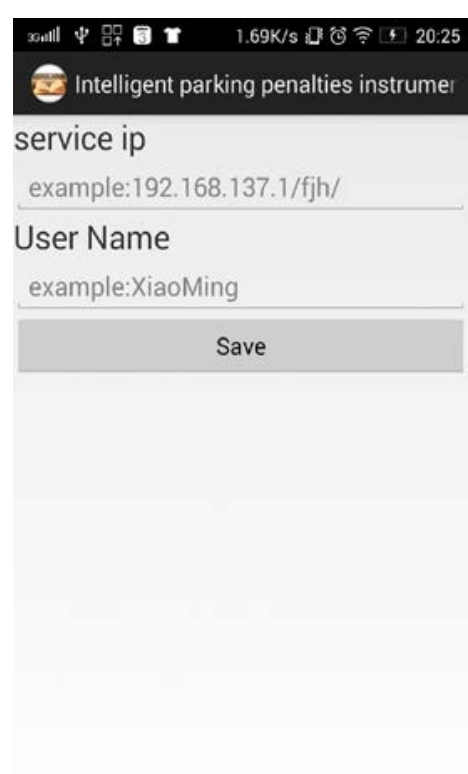

Fig.8 Parameter setting

\section{Conclusion}

This paper designs a penalty violation equipment based on Android. The equipment realizes taking photos of violation, license plate recognition, automatic positioning, automatic writing ticket and printing tickets on the spot by Bluetooth. The equipment using Smartphone technology and technology of mobile Internet applies to traffic enforcement in order to improve penalty efficiency. We have submitted applications for patents of invention for the research to State Intellectual Property Office. Proprietary names is that a method of parking violation penalty equipment. Application Number is 201510036469.8. Preliminary notice has been received. The patent has been in publication and substantive examination. The technique mentioned in the paper can be apply widely in traffic enforcement, urban management law enforcement, electrical circuit cruise, communications equipment inspection, etc.

\section{Acknowledgement}

In this paper, the research was sponsored by the Research Innovation Fund for College Students of Beijing University of Posts and Telecommunications (Project No. 2014-47).

\section{References}

[1] Qin Xiaowen, Wen Zhifang, Qiao Weiwei. Image processing based on OpenCV. Electronic Test, 2011, 07.

[2] QIU Li-ke, WANG Xiao-nian, ZHU Jin, JIANG Ping. Classification and Index of Voucher Based on Template Batch and Tesseract. Computer and Modernization, 2010, 07

[3] ZHANG Yang. Design and Implementation of the Recognition Application Based on Tesseract Engine. XIDIAN University, 2013.

[4] LUO Caifu. Research and Implementation of Bluetooth Communication System Based on Android. North China Electric Power University, 2013.

[5] GONG Chengying, XING Jinghong, HU Yinbao. Data communication of Android mobile terminal and PHP and MySQL based on JSON. Industrial Instrumentation \& Automation, 2013, 01. 